\title{
Der neue Staudamm von Marib (Republik Jemen)
}

\author{
"I hope your realm has reservoirs that are large and full of water, located in different parts of the land, so that agriculture does \\ not depend on the caprice of the Rain God."
}

Rishi Narada in: The Mahabharata

\section{Zusammenfassung}

Große Bewässerungsprojekte in Trockengebieten sollen einen Beitrag zur wirtschaftlichen Entwicklung und zur Lösung der Probleme im Zusammenhang mit dem Bevölkerungswachstum leisten. In Marib wurde 1984 bis 1986 ein neuer Staudamm gebaut, der auf eine jahrtausendealte Bewässerungstradition aufbauen sollte. Das ursprüngliche Ziel, das Angebot von Oberflächenwasser auf das ganze Jahr gleichmäßig zu verteilen, wurde verfehlt. Das Staudammprojekt ist bisher gescheitert, weil die Widersprüche zur bestehenden Sozialstruktur zu groß sind. Nach wie vor findet eine große Übernutzung der Grundwasservorräte statt, so daß bei gleichbleibender Entwicklung die Lebensgrundlage der Region, das Wasser, allmählich ausgehen wird

\section{Einleitung}

Im ariden Gürtel der Erde stellen Bewässerungsprojekte eine große Hoffnung für die wirtschaftliche Entwicklung einzelner Länder und Regionen dar. Die Trockengebiete nehmen etwa einen Drittel der Erdoberfläche ein, ihr Bevölkerungsanteil ist wesentlich kleiner. Gerade in diesem Raum findet in verschiedenen Regionen der Erde aber ein rasantes Bevölkerungswachstum statt, sei es durch natürliches Wachstum, sei es durch Zuwanderung. Man denke beispielsweise an Nordafrika und den Nahen Osten oder an den Westen der USA.

Bewässerungsprojekte in tropischen und subtropischen Regionen dienen in erster Linie zur Verbesserung der Nahrungsmittelerzeugung. Einerseits kann die Anbaufläche und Anbauzeit ausgedehnt werden, und andererseits wird durch ein zuverlässigeres Wasserangebot das Risiko von Dürren und Überschwemmungen verringert. So ist es nicht erstaunlich, daß in den letzten Jahrzehnten viele neue Projekte zur Erschließung oder Verbesserung des Wasserangebots entstanden sind. Die Argumentation ist immer ähnlich: In Libyen soll mit dem «Great Man-Made River» eine Selbstversorgung mit Nahrungsmitteln erreicht werden (NZZ 12./13.10.1991); in Ägypten soll durch die Erschließung der Grundwasservorkommen im «New Valley» in der westlichen Wüste neuer Lebensraum für eine Million Menschen geschaffen werden (BLISS 1989: 213), und, als letztes Beispiel, in Kalifornien werden Milliardenbeträge in den Bau neuer Reservoire investiert, zur Sicherstellung der Wasserversorgung im nächsten Jahrtausend für die südlichen Agglomeratio- nen und die Landwirtschaftsgebiete im Central Valley (WINDHORST 1994: 182 ff.).

All diese Projekte haben große ökologische Folgen und beeinflussen den Wasserhaushalt der ganzen Region. Zudem führen sie zu sozialen Veränderungen, weil die traditionellen Bewässerungssysteme als Teil des bestehenden sozialen Netzes den modernen, meist exogenen Systemen weichen müssen.

Häufig wird in der Diskussion um Bewässerung aber auch die politische Rolle von Großprojekten unterschätzt. Der Staat übernimmt als Betreiber der Bewässerungsanlagen die Kontrolle über die Wasserressourcen und damit das Schlüsselelement der regionalen Wirtschaft. Zusätzlich erreicht er eine Legitimation für weitere Eingriffe in das politische und soziale Umfeld.

Im Jemen nahm der Anteil der künstlich bewässerten Flächen in den letzten 20 Jahren stark zu, so daß heute nur noch etwa drei Viertel der kultivierten Agrarfläche im Regenfeldbau genutzt werden. Neben der traditionellen Flutbewässerung entlang der Wadis hat die ErschlieBung des Grundwassers eine immer größere Bedeutung. Seit 1970 wurden mehr als 25000 neue Brunnen gebohrt (REPUBLIC OF YEMEN 1991: II-3). Die unkontrollierte Verbreitung von Dieselpumpen führt zu einem raschen Absinken des Grundwasserspiegels. Im Einzugsgebiet von Sana'a, der Hauptstadt des Jemens, sinkt dieser etwa 3 Meter pro Jahr.

In der hier dargestellten Region Marib setzte diese Entwicklung etwas später ein, dafür mit stärkerer Intensität. Durch die hohe Aridität ist die Landwirtschaft vollkommen von künstlich zugeführtem Wasser abhängig. Vor der Einführung der Dieselpumpen wurde das Wasser mit Ablenkdämmen auf die Felder geleitet. Dieses Wasser reichte für die erwünschte wirtschaftliche Entwicklung der Region aber nicht aus, so daß vermehrt die Bewässerung mit Grundwasser angewendet wurde, mit ähnlichen Folgen wie in Sana'a. Das beschränkte Wasserangebot aus den Grundwasserreserven erforderte daher neue Lösungen, die erst mit dem Bau eines Staudammes hätten gefunden werden sollen.

Stefan Kohler, dipl. Geograph, Geographisches Institut der Universität Zürich Irchel, Winterthurerstr. 190, 8057 Zürich 


\section{Geographische Rahmenbedingungen}

Marib liegt auf der östlichen Seite des jemenitischen Hochlandes (Abb. 1). Da die Region sowohl während des Sommermonsuns als auch während des Frühjahrregens jeweils im Lee dieses Gebirgszuges liegt, beträgt die durchschnittliche jährliche Niederschlagsmenge unter $50 \mathrm{~mm}$. Für eine landwirtschaftliche Nutzung reicht dies nicht aus. Zweimal jährlich erreichen aber während der Regenfälle im Hochland große Wassermengen Marib. Diese Fluten stammen aus dem Einzugsgebiet des Wadi Adhanah, das mit einer Fläche von über $10000 \mathrm{~km}$ durchschnittlich pro Jahr 200 Mio. $\mathrm{m}^{3}$ Wasser nach Marib führt. Dieses Wasserangebot ist aber sehr unzuverlässig und unvorhersehbar, das zehnjährige Minimum liegt bei 60 Mio., das entsprechende Maximum bei 400 Mio. $\mathrm{m}^{3}$ (ELECTROWATT 1978: 9).

Der eigentliche Siedlungsraum von Marib erstreckt sich auf eine Fläche von etwa $400 \mathrm{~km}^{2}$. Dieser Raum liegt unterhalb einer Stelle, wo das Wadi Adhanah durch eine Klus eingeengt wird und dann in der Wüstenebene aus- läuft. Diese Lage relativiert die klimatisch bedingte natürliche Ungunst durch zwei Faktoren. Einerseits ist die Flut an der engsten Stelle der Klus relativ leicht regulierbar, und andererseits verursachte das Wadi ständige Sedimentablagerungen, die heute als ergiebige Grundwasserspeicher dienen.

Marib ist Hauptort der gleichnamigen Provinz. Diese ist in neun Distrikte aufgeteilt, von denen der Distrikt Marib in etwa der Bewässerungsoase im untersten Teil des Wadi Adhanah entspricht. Dieser zählte 197713500 Einwohner (GEISER \& STEFFEN 1979), neuere Zahlen stehen keine zur Verfügung. Die Bevölkerung hat seither stärker zugenommen als die jährlichen 3,1\% des Landesdurchschnittes, denn das Gebiet hat starke Entwicklungsimpulse in der Landwirtschaft, der Ölförderung und durch die Auflösung der Grenze zwischen dem ehemaligen Nordund Südjemen erfahren. Eine Bevölkerungszahl von 35 000-45 000 Einwohnern dürfte damit zu Beginn der 90er Jahre erreicht worden sein. Neben der Landwirtschaft hat sich bereits auch ein Dienstleistungssektor

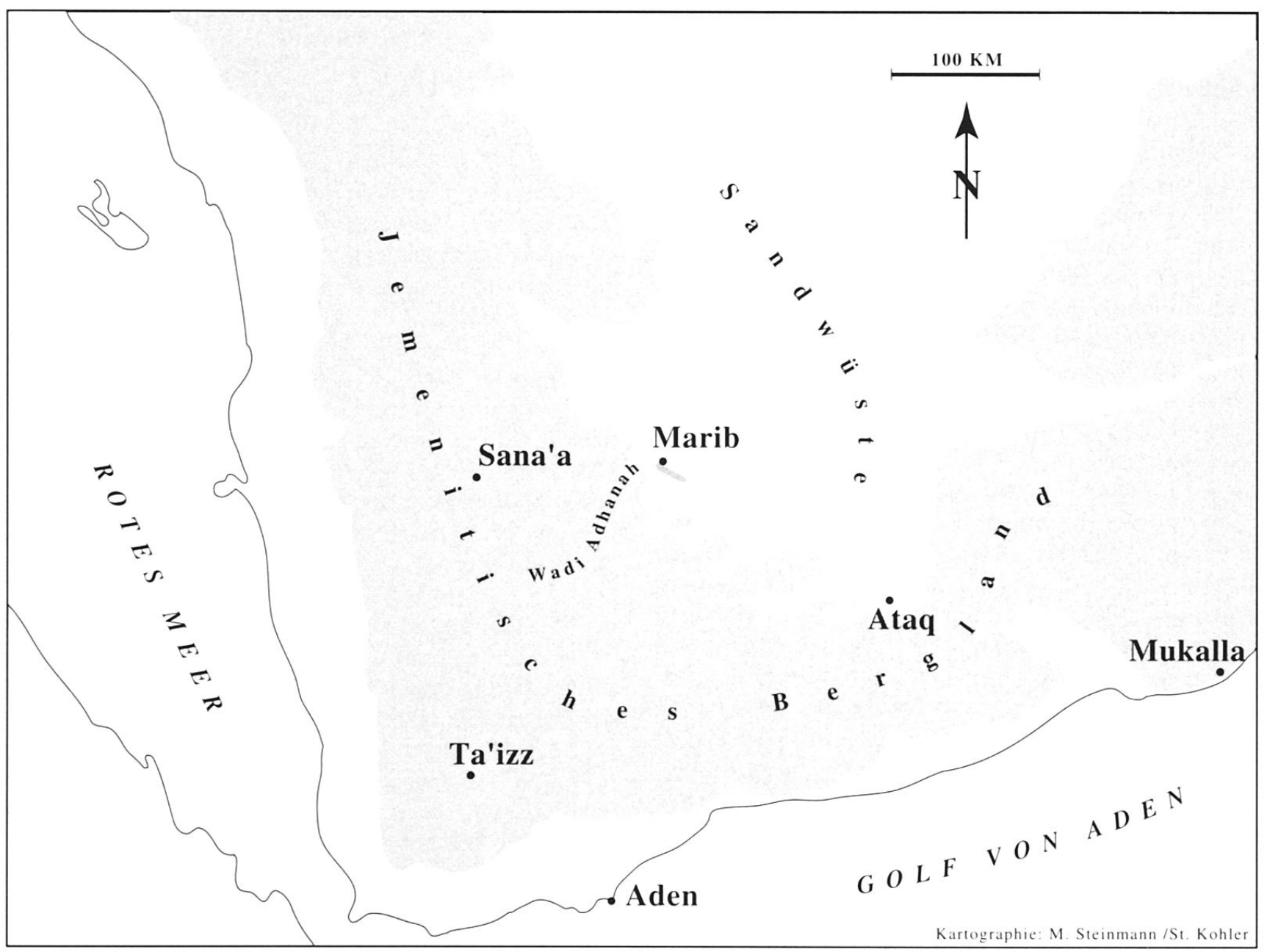

Abb.1 Ubersichtskarte von Südarabien. Marib liegt im ariden Innensaum des jemenitischen Hochlandes, während das Einzugsgebiet des Wadi Adhanah weit in dieses niederschlagsreichere Bergland reicht. 
herausgebildet, der von der zentralen Funktion von Marib profitiert.

Zudem hat der Bau der Teerstraße von Sana'a nach Marib 1980-1982 die relative Abgeschiedenheit der Region beendet. Dieser Bau ist im Zusammenhang mit anderen Infrastrukturbauten in der Umgebung zu sehen (Staudamm, Ölförderungsanlagen, Pipeline, neues Verwaltungszentrum und Hotels), aber auch mit dem Versuch des Staates, die traditionell geprägten politischen Strukturen im Osten des Landes näher an sich zu binden.

\section{Antike und traditionelle Wassernutzung}

In Marib geht der Beginn der geregelten und kontrollierten Bewässerung bis ins 3. Jahrtausend v. Chr. zurück. Während man zunächst nur das nach der Flut durchnäßte Wadibett nutzte, wurden die Nutzungssysteme mit der Zeit immer komplexer.

Während der Blütezeit des Sabäerreiches, also etwa zwischen 600 v. Chr. und 600 n. Chr., bestand der berühmte Ablenkdamm von Marib. Neben der hochentwickelten Bewässerungstechnik war die zentrale Stellung als Handelsmacht an der Weihrauchstraße für den sagenhaften Reichtum des Sabäerreiches verantwortlich.

Das damalige Bewässerungssystem bestand aus einem Damm, der $680 \mathrm{~m}$ lang war und an beiden Enden einen Auslaß hatte, von denen je ein Kanalsystem gespeist wurde (BRUNNER 1983: 96 f.). Aufgabe des Ablenkdammes war es, die in kurzer Zeit anfallende Wassermenge optimal zu nutzen. Die Dammanlage war so konstruiert, daß der Stauraum nur eine geringe Speicherkapazität hatte, dafür einen raschen Weitertransport des Wassers auf die Felder ermöglichte.

Dieses antike Bewässerungssystem ist nur im Zusammenhang mit den damaligen sozialen und politischen Verhältnissen zu verstehen. Die Wiederherstellung der Anlagen nach einem Dammbruch mußte möglichst rasch geschehen, um weitere, noch gravierendere Zerstörungen zu vermeiden. Dies konnte nur von einer starken Zentralmacht aus organisiert werden. Deshalb zerfiel während des politischen und wirtschaftlichen Niedergangs des Sabäerreiches zu Beginn des 7. Jh. n. Chr. auch das antike Bewässerungssystem.

Im Anschluß an diesen Zerfall und nach dem Exodus der Mehrzahl der Einwohner wurde auf einer wesentlich kleineren Fläche in Marib dezentrale Flutbewässerung betrieben, ähnlich wie in den anderen Tälern der Region. Der Unterschied zum antiken System bestand darin, daß früher von einem zentralen Damm aus ein großes, mit Kanälen verbundenes System bewässert wurde, während im späteren System viele kleinere dezentrale Dämme bestanden. Daran hat sich bis in die 80er Jahre nicht viel verändert. Die damit bewirtschaftete Fläche betrug 1973 etwa 3900 ha (SCHOCH 1978: 126) gegenüber etwa 9600 ha in der Antike (BRUNNER und HAEFNER 1992: $141)$.
Die dezentrale Flutbewässerung war den bestehenden sozialen und politischen Verhältnissen gut angepaßt. Die Sozialstruktur zeichnet sich durch die kleinen dezentralen Einheiten aus, die politische Organisation durch die hohe Autonomie der einzelnen Sippen. Jede dieser Einheiten kann nun mehrere kleinere Ablenkdämme unabhängig betreiben (KOHLER 1993: 83). Für den Unterhalt des Bewässerungssystems war somit keine übergeordnete politische Einheit nötig.

Erst der Bau des neuen Staudammes hat den Wasserhaushalt und damit die Kulturlandschaft wesentlich verändert.

\section{Der neue Staudamm}

Das Bedürfnis nach einer zuverlässigen, sich regenerierenden Wasserquelle stand in Marib bis Mitte der 80er Jahre der Tatsache gegenüber, daß eine periodische und starke Flut teilweise ungenutzt in die Wüste floß. Zudem wußte man vom antiken Ablenkdamm und seiner Bedeutung für die sabäische Hochkultur. Auf dieser Tradition aufbauend, wollte man wieder ein Bauwerk zur Begrünung der Oase von Marib erstellen.

Seit den 70er Jahren wurden Vorstudien zum Bau eines Staudammes ausgeführt, und 1984 erfolgte der erste Spatenstich. Der Bau des Dammes war ein Gemeinschaftswerk aus vier Nationen. Auftraggeber war der jemenitische Staat, gebaut wurde er von der türkischen Großbaufirma "Dogus Construction and Trading Co.", und die Zürcher Elektrowatt agierte als Generalplaner und überwachte die Bauarbeiten. Das ganze Projekt (Damm und Primärkanäle) wurde durch den «Entwicklungsfonds der Föderation der Arabischen Emirate» finanziert. Diese Entwicklungshilfe ist vor allem durch das persönliche Interesse des Staatsoberhauptes der Emirate, Scheich Zayed von Abu Dhabi, zu verstehen. Seine Vorfahren sollen einst in Marib gelebt und die Gegend im 7. Jh. verlassen haben, als der antike Damm endgültig brach.

Der Bau eines Stausees wurde gegenüber anderen Optionen vorgezogen, wobei folgende Argumentation jeweils für den Staudamm den Ausschlag gab (ELECTROWATT 1978: 27 ff.):

- Gegenüber dem bisherigen Zustand soll die von Elektrowatt vorgeschlagene Lösung zu einer Verbesserung sowohl der Arbeits- als auch der Wassereffizienz führen, da die bestehende Flutbewässerung zeitlich nur beschränkt nutzbar ist.

- Das Projekt einer verbesserten Flutbewässerung wurde aus denselben Gründen fallengelassen. Diskutiert wurden vier zentrale Ablenkdämme aus Beton, welche das Wasser je in ein Kanalsystem leiten sollten.

- Die Grundwassernutzung könnte kurz- und mittelfristig noch auf eine Fläche von 5500 ha ausgeweitet werden, von den 1978 bestehenden 1617 ha ausgehend. Trotz des guten Grundwasserspeichers sind die Vorräte aber limitiert, und zudem lohne sich der Einsatz von modernen Pumpen erst ab einer Betriebsgröße von 
10 bis 15 ha, was den bestehenden kleinflächigen Betrieben widerspreche.

Bereits die antike Anlage war an der günstigsten Stelle entstanden, und auch der neue Damm nutzt die enge Stelle in der obenerwähnten Klus aus. Das Wasser ist dort einfach zu fassen und in der weiter unten liegenden Ebene relativ einfach zu verteilen. Der neue Damm wurde allerdings etwa drei Kilometer oberhalb des alten Dammes errichtet. Damit wurde gewährleistet, daß die antiken Überreste der alten Anlage nicht beschädigt wurden.

Der Bau des Dammes sollte einen wichtigen Beitrag zur Erreichung der Ziele des damals laufenden Fünfjahresplans leisten, wozu explizit die Stärkung des Selbstversorgungsgrades mit Lebensmitteln erwähnt wurde (ELECTROWATt 1978: Annex III, 4). Im April 1986 war der neue Damm von Marib nach zwei Jahren Bauzeit fertiggestellt. Im Dezember des gleichen Jahres wurde er von den Präsidenten der Arabischen Emirate, der Türkei und Nordjemens eingeweiht. Die Gesamtkosten für den Damm und die Primärkanäle beliefen sich auf rund 75 Mio. US-\$, die zusätzlichen Kanäle für die Feinverteilung werden nochmals 25 Mio. kosten.

Die Dammauer hat eine Länge von $763 \mathrm{~m}$, eine Höhe von $38 \mathrm{~m}$ und staut einen See von maximal $30 \mathrm{~km}^{2}$ Fläche (vgl. Titelbild). Dabei wird ein Volumen von 400 Mio. m Wasser gestaut (ELECTROWATT 1978: 27 ff.).

Der neue Staudamm hat eine andere Funktion, als es die antike Anlage hatte. Während mit dem alten Damm das Wasser aus dem Wadibett ohne Verzögerung auf ein höheres Niveau und auf die Felder gebracht wurde, staut der neue Damm das Wasser und hat in der Mauer einen regulierbaren Auslaß, mit einer Kapazität von maximal $35 \mathrm{~m}^{3} / \mathrm{sec}$. Anlagen zur Gewinnung elektrischer Energie wurden keine eingebaut.

Nach dem Auslaß fließt das Wasser wieder im alten Wadibett (Abb. 2) und sollte in vier gestaffelten Fassungen nach 5, 9, 14 und $17 \mathrm{~km}$ ein Primärkanalsystem speisen, um jeweils 157 ha, 145 ha, 487 ha und 5515 ha Fläche zu bewässern. Die ersten drei dieser Fassungen bestehen nur aus Dämmen und Schleusen, womit das Wasser direkt in die Kanäle abgelenkt würde, während für die vierte Fassung infolge der Größe ihrer zu bewässernden Fläche noch ein Zwischenbecken mit der Kapazität eines Tagesverbrauchs gebaut wurde. Von den vier Fassungen im Wadibett soll das Wasser in Betonkanälen zu sekundären Schleusen gebracht werden. Von dort ist die Weiterführung und die Kontrolle des Wassers Sache der einzelnen Wasserverbraucher.

Die Bewässerungsmethoden sollten sich durch diese Verteilung so wenig wie möglich von den damals bestehenden unterscheiden, sowohl von der technischen als auch von der institutionellen Seite her. Die Planung durch die Elektrowatt endet dort, wo der Hauptkanal in das sekundäre System mündet. Da die Feinverteilung vom sekundären Kanalsystem abwärts in der Verantwortung der Wasserverbraucher liegt, sollte den traditionellen Stammesstrukturen weitgehend entgegengekommen werden.

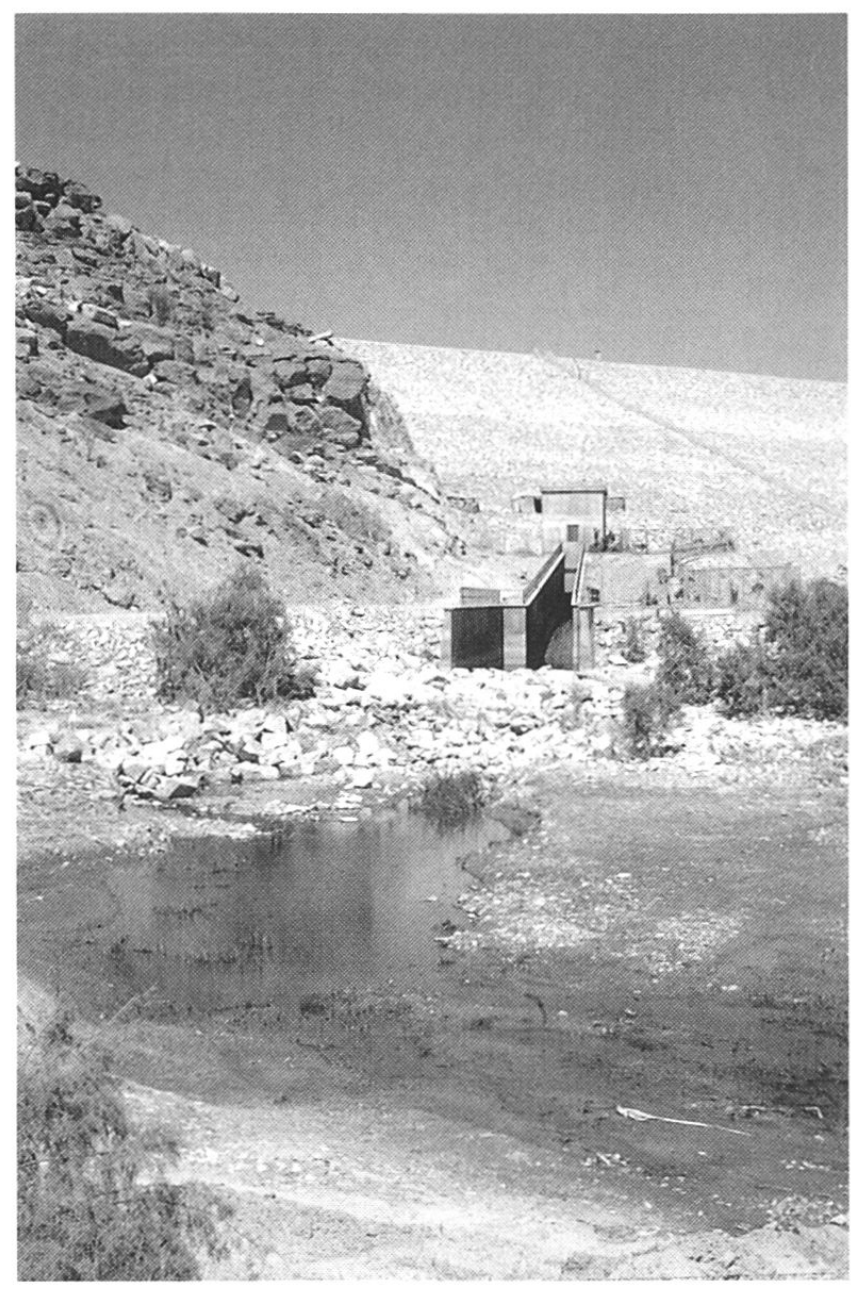

Abb.2 Wasserauslaß in der Staumauer (Foto: Stefan Kohler)

Ein zweiter wichtiger Aspekt des Projektes ist der Miteinbezug der Grundwassernutzung. Sowohl aus dem Seebecken als auch aus dem Wadibett wird das Grundwasser für die Region Marib stärker angereichert, als wenn der Sayl in die Wüste ausläuft. Der Damm und der offene Abfluß nach dem Auslaß bewirken eine ständige Anreicherung des Grundwassers. Auch dies wird als Argument für die Unterstützung der damaligen dezentralen Wassernutzung verstanden.

\section{Auswirkungen}

Der Begriff «Auswirkung» ist ursprünglich ein wertfreier Ausdruck, im Zusammenhang mit größeren Infrastrukturprojekten ist er aber heute meist mit negativen Assoziationen verbunden. Allfällige positive Folgen werden als selbstverständlich betrachtet und kaum speziell erwähnt. In Marib fällt es jedoch schwer, positive Seiten des 
neuen Dammes zu würdigen, denn bis heute bestehen die Grundprobleme im Zusammenhang mit der Wassernutzung nach wie vor, während sich neue soziale und ökologische Probleme ergeben haben.

Der Bau des Staudammes hat in der Region Marib den Wasserhaushalt und damit die Natur- und Kulturlandschaft stark verändert. Die ursprüngliche Idee war die Verteilung des Wasserangebotes auf das ganze Jahr. Dieses Ziel wurde bisher nicht erreicht, und offensichtliche Mängel in der Konzeption deuten darauf hin, daß auch in Zukunft kaum wesentliche Verbesserungen stattfinden werden. Tatsächlich fließt seit der Eröffnung des Dammes erst durch die oberste der vier Fassungen etwas Wasser, um damit Felder zu bewässern. Demgegenüber konnte die Anreicherung des Grundwassers etwas verbessert werden, weil mehr Wasser im oberen Teil der Region versickert und damit weiter unten nochmals zur Verfügung steht. Trotzdem sinkt der Grundwasserspiegel um etwa 1,5 Meter pro Jahr (REPUBLIC OF YEMEN 1991: II-4). Die Versickerung des Grundwassers aus dem Seebecken wird allerdings allmählich schwinden, weil der Seeboden durch die ständige Sedimentierung immer wasserundurchlässiger wird.

Der Grund für das Scheitern des Staudammes und damit für die Unfähigkeit, das Seewasser für die Bewässerung zu nutzen, liegt an der mangelnden Übereinstimmung des Projektes mit den sozialen und politischen Realitäten der Region. Die vermeintliche «Wiedererstehung des antiken Dammes", wie der neue Staudamm zunächst gepriesen wurde (NZZ: 2./3.4. 1988), ist bisher nicht gelungen, weil die politischen Rahmenbedingungen nicht mehr die gleichen sind wie in der Antike. Der jemenitische Staat hat den Bau wesentlich gefördert und zeigte damit sein Interesse, den traditionell eher autonomen Osten stärker an sich zu binden.

In einer Stellungnahme von Elektrowatt werden die institutionellen Probleme für das Scheitern verantwortlich gemacht: «... Das Projekt mußte vom Kunden [Föderation der Arabischen Emirate] unvollendet abgebrochen werden, weil es der Regierung nicht gelang, das für den Bau der weiteren Kanäle notwendige Land ordnungsgemäß von den Eigentümern zu erwerben» (NZZ: 25.2.1992).

Dies zeigt, daß der Staudamm mehr ist als nur ein Bewässerungsprojekt. In der Hand seiner Betreiber bedeutet er ein Druckmittel, das die Region in ihrem wichtigsten Lebensbereich, dem Wasser, abhängig macht. Ein Beispiel für den Stolz der Zentralregierung auf den neuen Staudamm ist die Darstellung des Bauwerkes als Symbol des fortschrittlichen Jemens an verschiedenen Stellen. So präsentiert sich der Damm auf einem Propagandaplakat auf dem Hauptplatz in Sana'a (Abb. 3) oder auf der Rückseite der 10-Rial-Banknote.

Der Sedimenteintrag in den See beträgt etwa 2,5 Mio. $\mathrm{m}^{3}$ pro Jahr (ELECTROWATT 1981: 9). Damit wird der See in 200 Jahren zusedimentiert sein, in 100 Jahren wird er seine Funktion bereits nicht mehr erfüllen können. Zudem fehlt die Sedimentfracht auf den Feldern als natürlicher
Dünger. Das bedeutet gegenüber der bisherigen Flutbewässerung, daß nun Kunstdünger zugekauft werden muß.

Neben all diesen Problemen ist in der Region eine bisher eher unbekannte Gefahr aufgetaucht, nämlich Krankheiten, deren Zwischenwirte an stehendes Wasser gebunden sind. Dazu gehören Bilharziose, Onchozerkose und Malaria. Durch das gestaute Wasser, aber auch durch die ständig feuchte Landschaft unterhalb des Dammes sind für diese Krankheiten ideale Lebensbedingungen geschaffen worden.

\section{Ausblick}

Der Staudamm von Marib wurde gebaut, um die beiden Grundprobleme der Wassernutzung in dieser Region zu lösen: das nur periodisch vorhandene Wasserangebot und die Übernutzung der Grundwasservorkommen.

Trotz der erwähnten Probleme des Staudammprojektes hat in Marib die landwirtschaftlich genutzte Fläche in den vergangenen Jahren stark zugenommen. Dies hat zur noch stärkeren Beanspruchung des Grundwasservorrats geführt, so daß bei gleichbleibendem Verbrauch dessen obere Schichten noch etwa 20 bis 40 Jahre reichen werden. Ein sinkender Grundwasserspiegel bedeutet auch einen höheren Salzgehalt des verbleibenden Wassers, damit erhöht sich die Gefahr der Versalzung der damit bewässerten Böden.

Zudem wird durch das ständige Absinken des Grundwasserspiegels die Nutzung immer aufwendiger, weil tiefere Brunnen gebohrt und leistungsfähigere Dieselpumpen installiert werden müssen und der Energieaufwand für die Hebung des Wassers höher wird. Da sich diese Kosten nicht mehr alle Bauern leisten können, ist zu erwarten, daß sich der Grundwasserspiegel auf einem tieferen Niveau einpendeln wird. Dann wird nur noch ein Teil der heute bebauten Fläche nutzbar sein, und viele der Bauern, die zum Teil erst in den letzten Jahren zugezogen sind, werden ihre Lebensgrundlage verlieren.

Diese Entwicklung kann durch die Erschließung neuer Grundwasservorkommen im Nordosten der Region verlängert werden. Weil dieses Grundwasser sich nur sehr langsam regeneriert, wäre die Gefahr der Übernutzung hier besonders groß. Zudem würde die Erschließung dieser Quellen wieder ein Großprojekt bedeuten, mit ähnlichen sozialen und politischen Widerständen wie beim neuen Staudamm.

Besser als die Erschließung neuer Wasservorkommen sind Maßnahmen, um die bestehenden Ressourcen effizienter zu nutzen. Auf der nationalen planerischen Ebene ist dafür eine sorgfältige Beobachtung über die vorhandenen Ressourcen nötig. Aus diesem Wissen heraus soll ein neues, der modernen Nutzung angepaßtes Wasserrecht erarbeitet werden, das auf die ökologischen und ökonomischen Auswirkungen ausgerichtet ist. Vorschlä- 


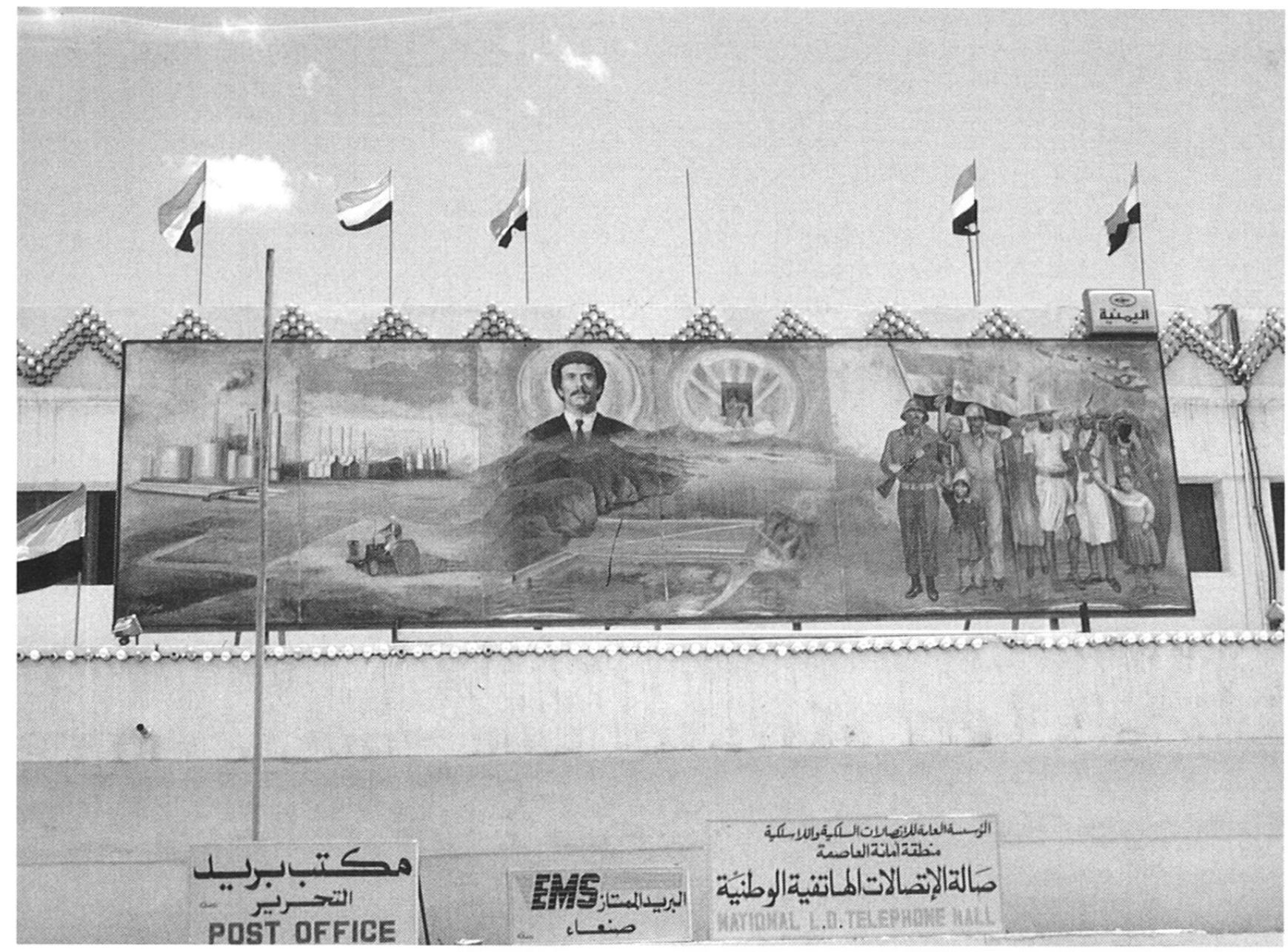

Abb.3 Der jemenitische Entwicklungsweg auf einem Plakat über dem Hauptpostamt in Sana'a (von rechts nach links): Nach der Revolution von 1962 folgt die landwirtschaftliche Entwicklung, symbolisiert durch den neuen Staudamm von Marib und einen Traktor. Am Schluß steht die Industrialisierung durch die Ölwirtschaft. Über allem stehen das Symbol der Vereinigung Nord- und Südjemens und der Staatspräsident Ali Abdallah Saleh (Foto: Stefan Kohler).

ge dafür wurden in einem Bericht formuliert, der in $\mathrm{Zu}$ sammenarbeit des Planungsministeriums mit dem UNDP entstanden ist (REPUBLIC OF YEMEN 1991: II-8.). Einer der Hauptpunkte dieses Berichtes ist die Neuorganisation der für die Wassernutzung zuständigen Ministerien und Ämter, die bis anhin noch schlecht koordiniert waren und deshalb keine nationale Strategie zur nachhaltigen Nutzung aufstellen konnten.

Konkrete Schritte, wie die Situation verbessert werden könnte, fehlen im Gesetzesvorschlag weitgehend. Einzig die Übernahme der im früheren Südjemen üblichen Bewilligungspflicht für Grundwasserbohrungen soll im ganzen Land eingeführt werden. Weitere Restriktionen sind aber nötig, um das Ziel einer ausgeglichenen Wasserbilanz zu erreichen.

Derartige Eingriffe bedeuten einschneidende Veränderungen der rechtlichen und politischen Strukturen und damit auch der sozialen Verhältnisse. Die Schwierigkei- ten, die damit verbunden sein dürften, sind groß, wie das Scheitern des neuen Staudammes gezeigt hat. Angesichts der Tatsache, daß die Verknappung der Wasserressourcen am Schluß auch zu weitgehenden Beeinträchtigungen der traditionellen Strukturen führt durch die Verschlechterung ihrer Lebensgrundlage, sind diese Maßnahmen aber auf jeden Fall gerechtfertigt.

\section{Literatur}

BLISS, F. (1989): Traditionelle Bewässerung und moderne Regionalentwicklung; Das ägyptische Beispiel "Neues Tal». In: Die Erde, Nr. 120, 213-221

BRUNNER, U. (1983): Die Erforschung der antiken Oase von Marib mit Hilfe geomorphologischer Untersuchungsmethoden, Diss., Zürich. 
BRUNNER, U., \& HAEFNER, H. (1990): Altsüdarabische Bewässerungsoasen. In: Die Erde, Nr. 121, 135-153.

ELECTROWATT Engineering Services Ltd (1978): Marib Dam and Irrigation Project, Main Report und Annexes I-XII, Zürich.

- (1981): Marib Dam and Irrigation Project, Engineer Report, Volume 6, Project Description, Zürich.

GEISER, U., \& STEFFEN, H. (1977): Population Distribution, Administrative Division and Land Use in the Yemen Arab Republic, Karte 1:500 000, Zürich.

KOHLER, S. (1993): Wassernutzung im ariden Osten des Jemens; eine Bewertung von verschiedenen Bewässerungssystemen im Wadi Marcha und Marib mit Hilfe des Konzepts der Nachhaltigkeit, Diplomarbeit am Geographischen Institut der Universität Zürich.
NZZ, Neue Zürcher Zeitung, Zürich, diverse Ausgaben.

REPUBLIC OF YEMEN (1991), Ministry of Planning \& Development and UNDP DTCD: Aide Memoire Water Sector Overview, Sana'a.

SCHOCH, R. (1978): Die antike Kulturlandschaft des Stadtbezirkes Saba' und die heutige Oase von Marib in der Arabischen Republik Jemen. In: Geographica Helvetica, Nr. 3/33, $121-129$.

WINDHORST, H.-W. (1994): Wasser für Millionen. Der Metropolitan Water District of Southern California. In: KLOHN W., \& WINDHORST, H.-W. (Hrsg.): Bewässerungslandwirtschaft in Kalifornien unter Dürrestreß, 177-190, Vechta.

Der Autor dankt der "Stiftung für wissenschaftliche Forschung an der Universität Zürich" für die Finanzierung der Reisekosten für den Feldaufenthalt im Mai/Juni 1992. 